\title{
Some New Classes of Heronian Mean Graphs
}

\section{S.S. Sandhya}

Department of Mathematics, Sree Ayyappa College for Women,

\section{E.Ebin Raja Merly}

Department of Mathematics, Nesamony Memorial Christian College,

\section{S.D.Deepa}

Research Scholar, Nesamony Memorial Christian College,

Chunkankadai, Tamilnadu, India. Marthandam, Tamilnadu, India. Marthandam, Tamilnadu, India.

\begin{abstract}
In this paper, We discuss Heronian Mean labeling behavior of Caterpillar, Shadow graph, Middle graph, Total graph, Splitting graph and Duplication of a vertex. Also We investigate Heronian Mean labeling of Wheel Graph.
\end{abstract}

Keywords: Heronian Mean labeling, Shadow graph, Middle graph, Total graph, Splitting graph, Duplication, Wheel Graph.

AMS Subject Classification: 05C78

\section{INTRODUCTION}

The graph considered here will be simple, finite and undirected graph without loops or parallel edges. For all detailed survey of graph labeling, we refer to J.A.Gallian [1]. Terms not defined here are used in the sense of Harary [2].

We shall make frequent references to the following definitions

\section{Definition 1.1:}

A graph $\mathbf{G}=(\mathbf{V}, \mathbf{E})$ with $\mathrm{p}$ vertices and $\mathrm{q}$ edges is said to be a Heronian Mean graph if it is possible to label the vertices $\mathbf{x} \in \mathbf{V}$ with distinct labels $\mathbf{f}(\mathbf{x})$ from $\mathbf{1}, \mathbf{2}, \ldots, \mathbf{q}+\mathbf{1}$ in such a way that when each edge $\mathbf{e}=\mathbf{u v}$ is labeled with,

$$
\mathbf{f}(\mathbf{e}=\mathbf{u v})=\left\lceil\frac{\mathbf{f}(\mathbf{u})+\sqrt{\mathbf{f}(\mathbf{u}) \mathbf{f}(\mathbf{v})}+\mathbf{f}(\mathbf{v})}{3}\right\rceil(\mathbf{O R})\left\lfloor\frac{\mathbf{f}(\mathbf{u})+\sqrt{\mathbf{f}(\mathbf{u}) \mathbf{f}(\mathbf{v})}+\mathbf{f}(\mathbf{v})}{3}\right\rfloor
$$

then the edge labels are distinct. In this case $\mathbf{f}$ is called a Heronian Mean labeling of G.

\section{Definition 1.2:}

A tree which yields a path when its pendant vertices are removed is called a Caterpillar

\section{Definition 1.3:}

Let $\mathrm{G}$ be a connected graph and $G^{\prime}$ be the copy of $\mathrm{G}$. Then Shadow graph $D_{2}(G)$ is obtained by joining each vertex $u$ in $\mathrm{G}$ to the neighbours of the corresponding vertex $u^{\prime}$ in $G^{\prime}$.

\section{Definition 1.4:}

The Middle graph $M(G)$ of a graph $G$ whose vertex set is $V(G) \cup E(G)$ and in which two vertices are adjacent if and only if either they are adjacent edges of $G$ (or) one is a vertex of $G$ and the other is an edge incident on it.

\section{Definition 1.5:}

The Total graph $T(G)$ of graph $G$ is the graph whose vertex set is $V(G) \cup E(G)$ and two vertices are adjacent whenever they are either adjacent (or) incident in $\mathrm{G}$. 


\section{Definition 1.6:}

The Splitting graph $S^{\prime}(G)$ is obtained by adding new vertex $v^{\prime}$ corresponding to each vertex $v$ of $\mathrm{G}$ such that $\mathrm{N}(v)=\mathrm{N}\left(v^{\prime}\right)$, Where $\mathrm{N}(v)$ and $\mathrm{N}\left(v^{\prime}\right)$ are the neighbourhood sets of $v$ and $v^{\prime}$ resp.

\section{Definition 1.7:}

Duplication of a vertex $v_{k}$ by a new edge $e=v_{k}{ }^{\prime} v_{k}$ " in a graph G produces a new graph $G$ such that $\mathrm{N}\left(v_{k}{ }^{\prime}\right) \cap \mathrm{N}\left(v_{k}{ }^{\prime \prime}\right)=v_{k}$.

The notion of Heronian Mean labeling was introduced by S.S.Sandhya, E.Ebin Raja Merly and S.D.Deepa and the Heronian Mean labeling behavior of Path $P_{n}$, Cycle $C_{n}$, Star $\mathrm{K}_{1, \mathrm{n}}$, Triangular Snake $T_{n}$, Quadrilateral Snake $Q_{n}$, Comb , ladder $\boldsymbol{L}_{\boldsymbol{n}}$, Step Ladder, Crown, Complete graph has been investigated in [5] and [6]. In this paper we contribute some new results for Heronian Mean labeling of graphs.

\section{Main Results}

\section{Theorem: 2.1}

Let $\mathrm{G}$ be a graph obtained by attaching pendent edges to both sides of each vertex of a path $P_{n}$. Then $G$ is a Heronian mean graph.

\section{Proof:}

Consider a graph $\mathrm{G}$ which is obtained by attaching pendant edges to both sides of each vertex of a path $\mathrm{P}_{\mathrm{n}}$. Let $\mathrm{P}_{\mathrm{n}}$ be a path $\mathrm{u}_{1}, \mathrm{u}_{2}, \mathrm{u}_{3}, \ldots \ldots \mathrm{u}_{\mathrm{n}}$. Let $\mathrm{v}_{\mathrm{i}}$ and $\mathrm{w}_{\mathrm{i}}$ be the pendant vertices adjacent to $\mathrm{u}_{\mathrm{i}}, 1 \leq i \leq \mathrm{n}$.

Define a function $f: V(G) \rightarrow\{1,2,3, \ldots, q+1\}$ by $f\left(u_{i}\right)=3 i-2,1 \leq i \leq n$.

$$
\begin{aligned}
& f\left(v_{i}\right)=3 i-1, \quad 1 \leq i \leq n . \\
& f\left(w_{i}\right)=3 i, 1 \leq i \leq n .
\end{aligned}
$$

Edges are labeled with, $f\left(u_{i} u_{i+1}\right)=3 i, 1 \leq i \leq n-1$

$$
\begin{aligned}
& f\left(u_{i} v_{i}\right)=3 i, 1 \leq i \leq n \\
& f\left(u_{i} w_{i}\right)=3 i-1,1 \leq i \leq n
\end{aligned}
$$

Hence $\mathrm{G}$ is a Heronian mean graph.

Example 2.2: A Heronian mean labeling of $G$ with 15 vertices and 14 edges is given below

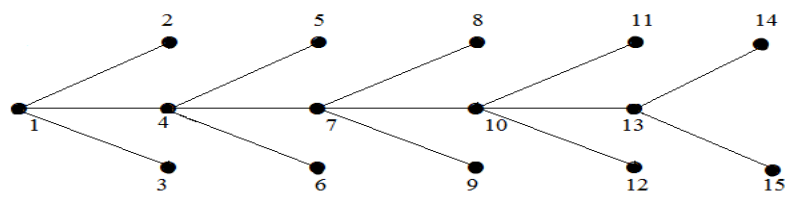

Figure:1

\section{Theorem: 2.3}

Let $G$ be a graph obtained by attaching paths of length $0,1,2, \ldots \ldots, n-1$ on both sides of each vertex of $P_{n}$, then $G$ is a Heronian mean graph.

\section{Proof:}

Let $G$ be a graph obtained by attaching paths of length $0,1,2, \ldots \ldots . n-1$ on both sides of each vertex of $P_{n}$. Let $u_{11} u_{22} \ldots \ldots u_{n n}$ are the vertices of $P_{n}$.

Define a function, $f: V(G) \rightarrow\{1,2,3, \ldots ., q+1\}$ by

$$
f\left(u_{i j}\right)=(i-1)^{2}+j, 1 \leq i \leq n, 1 \leq j \leq 2 i-1
$$

From the above labeling pattern, we get distinct edge labels.

Thus $f$ provides a Heronian mean labeling. 


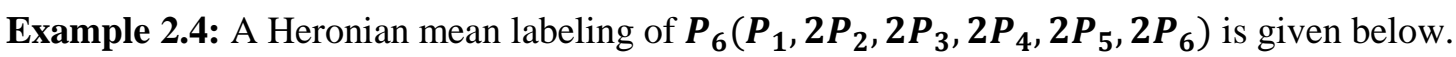

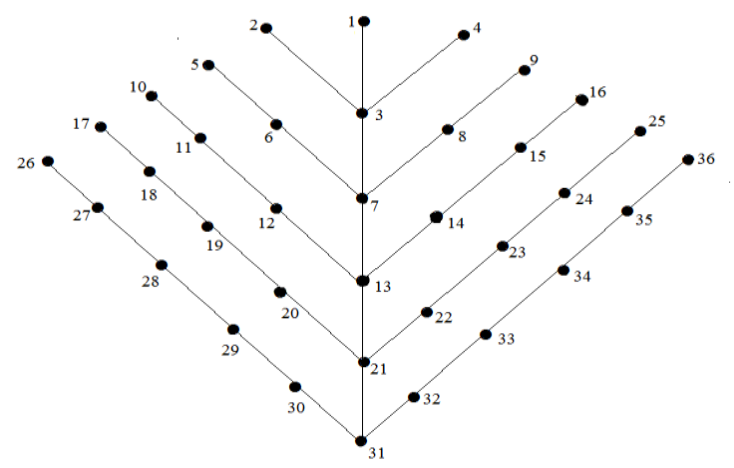

Figure:2

\section{Theorem: 2.5}

The graph $D_{2}\left(P_{n}\right)$ is a Heronian mean graph.

\section{Proof:}

Let $u_{1} u_{2} \ldots \ldots u_{n}$ be the vertices of a Path $P_{n}$ and $v_{1} v_{2} \ldots \ldots v_{n}$ be the newly added vertices corresponding to the vertices $u_{1} u_{2} \ldots \ldots u_{n}$ in order to obtain $D_{2}\left(P_{n}\right)$. Denoting $G=D_{2}\left(P_{n}\right)$, then $|V(G)|=2 n$ and $|E(G)|=4(n-1)$.

Define a function, $f: V(\boldsymbol{G}) \rightarrow\{1,2, \ldots . q+1\}$ by $f\left(u_{1}\right)=1, f\left(u_{2}\right)=3$,

$$
\begin{gathered}
f\left(u_{i}\right)=4(i-1), 3 \leq i \leq n, \\
f\left(v_{i}\right)=\left\{\begin{array}{c}
4 i-2,1 \leq i \leq n-1, \\
4 i-3, i=n .
\end{array}\right.
\end{gathered}
$$

Edges are labeled with $f\left(u_{i} u_{i+1}\right)=4 i-3,1 \leq i \leq n-1$,

$$
\begin{aligned}
& f\left(v_{i} v_{i+1}\right)=4 i, 1 \leq i \leq n-1, \\
& f\left(u_{i} v_{i+1}\right)=\left\{\begin{array}{c}
4 i-1, i=1 \\
4 i-2,2 \leq i \leq n-1,
\end{array}\right. \\
& f\left(v_{i} u_{i+1}\right)=\left\{\begin{array}{c}
4 i-2, i=1 \\
4 i-1,2 \leq i \leq n-1 .
\end{array}\right.
\end{aligned}
$$

Hence $D_{2}\left(P_{n}\right)$ is a Heronian Mean graph.

Example 2.6: Shadow graph of Path $P_{5}$ and its Heronian mean labeling is shown in figure:3.

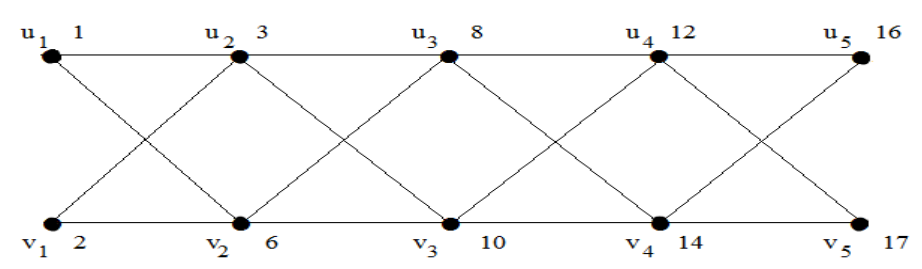

Figure:3

\section{Theorem: 2.7}

Middle graph of Path $P_{n}$ is a Heronian mean graph.

Proof:

Let $u_{1} u_{2} \ldots \ldots u_{n}$ be the vertices and $e_{1} e_{2} \ldots \ldots e_{n-1}$ be the edges of Path $P_{n}$ and $G=$ $M\left(P_{n}\right)$ be the Middle graph of Path $P_{n}$. Here $|V(G)|=2 n-1$ and $|E(G)|=3 n-4$.

Define a function $f: V(G) \rightarrow\{1,2,3, \ldots, q+1\}$ by, $\left(u_{1}\right)=1, f\left(u_{i}\right)=3 i-3,2 \leq i \leq n$.

$$
f\left(e_{i}\right)=3 i-1,1 \leq i \leq n-1 \text {. }
$$


Edges are labeled with $f\left(u_{i} e_{i}\right)=3 i-2,1 \leq i \leq n-1$,

$$
\begin{aligned}
& f\left(u_{i} e_{i-1}\right)=3 i-1,2 \leq i \leq n-1, \\
& f\left(e_{i} e_{i+1}\right)=3 i, 1 \leq i \leq n-2 .
\end{aligned}
$$

Hence $M\left(P_{n}\right)$ is a Heronian Mean graph.

Example 2.8: Middle graph of Path $P_{5}$ and its Heronian mean labeling is shown in figure:4.

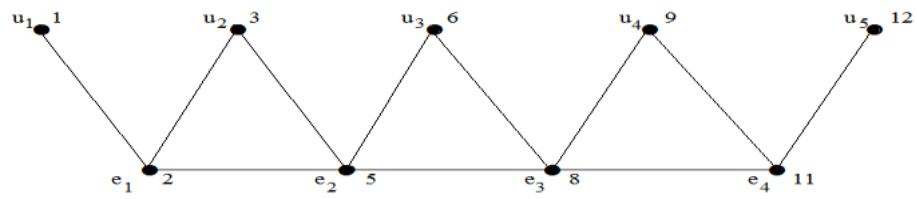

Figure:4

\section{Theorem: 2.9}

Total graph of Path $P_{n}$ is a Heronian mean graph.

\section{Proof:}

Let $u_{1} u_{2} \ldots \ldots u_{n}$ be the vertices and $\quad e_{1} e_{2} \ldots \ldots e_{n-1}$ be the edges of Path $P_{n}$ and $G=$ $T\left(P_{n}\right)$ be the Total graph of Path $P_{n}$. Here $|V(G)|=2 n-1$ and $|E(G)|=4 n-5$.

Define a function $f: V(G) \rightarrow\{1,2,3, \ldots, q+1\}$ by $f\left(u_{1}\right)=1, f\left(u_{i}\right)=4 i-4,2 \leq i \leq n$.

$$
f\left(e_{1}\right)=3, f\left(e_{i}\right)=4 i-2,2 \leq i \leq n-1 .
$$

Edges are labeled with $f\left(u_{i} u_{i+1}\right)=4 i-2,1 \leq i \leq n-1$

$$
\begin{aligned}
& f\left(u_{i} e_{i}\right)=4 i-3,1 \leq i \leq n-1 \\
& f\left(u_{i} e_{i-1}\right)=4 i-1,2 \leq i \leq n, \\
& f\left(e_{i} e_{i+1}\right)=4 i, 1 \leq i \leq n-2 .
\end{aligned}
$$

Hence $T\left(P_{n}\right)$ is a Heronian Mean graph.

Example 2.10: Total graph of Path $P_{6}$ and its Heronian mean labeling is shown in figure:5.

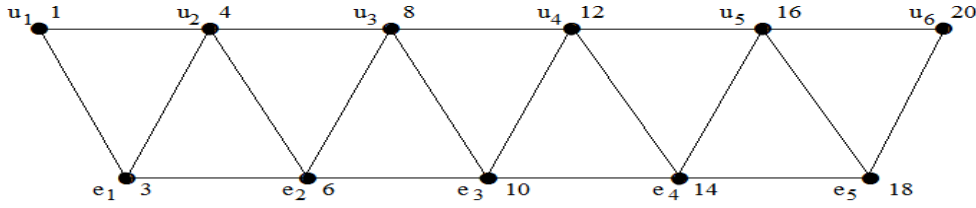

Figure:5

\section{Theorem: 2.11}

Splitting graph of Path $P_{n}$ is a Heronian mean graph.

\section{Proof:}

Let $u_{1} u_{2} \ldots \ldots u_{n}$ be the vertices and $e_{1} e_{2} \ldots \ldots e_{n-1}$ be the edges of Path $P_{n}$. Let $v_{1} v_{2} \ldots \ldots v_{n}$ be the newly added vertices to form the Splitting graph of Path $P_{n}$. Here $|V(G)|=2 n$ and $|E(G)|=3 n-3$.

Define a function $f: V(G) \rightarrow\{1,2,3, \ldots, q+1\}$ by $\left(u_{1}\right)=2, f\left(u_{i}\right)=3 i-3,2 \leq i \leq n$.

$$
f\left(v_{i}\right)=3 i-2,1 \leq i \leq n \text {. }
$$

Edges are labeled with, $f\left(v_{i} u_{i+1}\right)=\left\{\begin{array}{c}3 i-2, i=1 \\ 3 i-1,2 \leq i \leq n-1 \text {, }\end{array}\right.$ 


$$
\begin{gathered}
f\left(v_{i} v_{i+1}\right)=\left\{\begin{array}{c}
3 i-1, i=1 \\
3 i, 2 \leq i \leq n-1
\end{array}\right. \\
f\left(v_{i} u_{i-1}\right)=\left\{\begin{array}{c}
3 i-3, i=2 \\
3 i-5,3 \leq i \leq n-1,
\end{array}\right.
\end{gathered}
$$

Hence $S^{\prime}\left(P_{n}\right)$ is a Heronian Mean graph.

Example 2.12: Splitting graph of Path $P_{6}$ and its Heronian mean labeling is shown in figure:6.

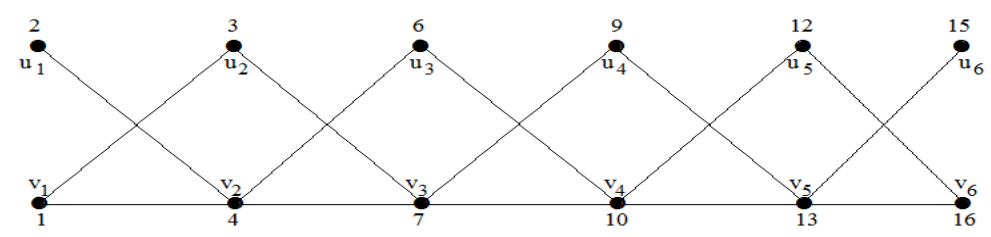

Figure:6

\section{Theorem: 2.13}

Duplicating each vertex by an edge in Path $P_{n}$ is a Heronian mean graph.

\section{Proof:}

Let $u_{1} u_{2} \ldots \ldots u_{n}$ be the vertices of a Path $P_{n}$. Let $\mathrm{G}$ be the graph obtained by duplicating each vertex $v_{i}$ of $P_{n}$ by an edge $v_{i}{ }^{\prime}, v_{i}{ }^{\prime \prime}$ at a time, $1 \leq i \leq n$. Here $|V(G)|=3 n$ and $|E(G)|=4 n-1$.

Define a function $f: V(G) \rightarrow\{1,2,3, \ldots, q+1\}$ by $f\left(u_{i}\right)=4 i-2,1 \leq i \leq n$,

$$
\begin{aligned}
& f\left(v_{i}^{\prime}\right)=4 i-3,1 \leq i \leq n, \\
& f\left(v_{i}^{\prime \prime}\right)=4 i-1,1 \leq i \leq n,
\end{aligned}
$$

Edges are labeled with $f\left(u_{i} u_{i+1}\right)=4 i, 1 \leq i \leq n-1$,

$$
\begin{gathered}
f\left(u_{i} v_{i}^{\prime}\right)=4 i-3,1 \leq i \leq n, \\
f\left(u_{i} v_{i}^{\prime \prime}\right)=4 i-1,1 \leq i \leq n, \\
f\left(v_{i}^{\prime} v_{i}^{\prime \prime}\right)=4 i-2,1 \leq i \leq n,
\end{gathered}
$$

Hence duplicating each vertex by an edge in Path $P_{n}$ is a Heronian Mean graph.

Example 2.14: Duplicating each vertex by an edge in Path $P_{4}$ and its Heronian mean labeling is shown in figure: 7 .

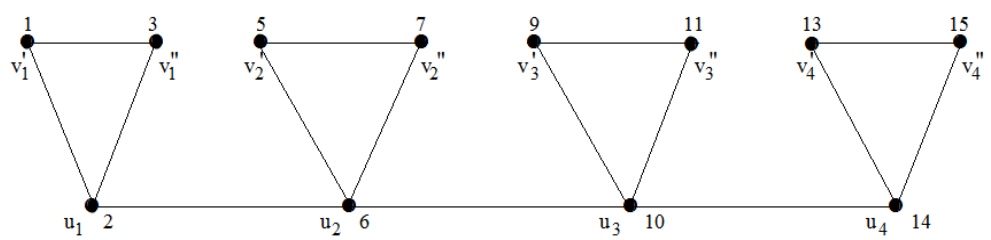

Figure:7

\section{Theorem: 2.15}

For $\mathbf{n}>4$, Wheel Graph $W_{n}$ is not a Heronian mean graph.

\section{Proof:}

For $n=3$ and $n=4$ clearly $W_{3}$ and $W_{4}$ are Heronian mean graphs. The labeling pattern of $W_{3}$ and $W_{4}$ is shown below. 

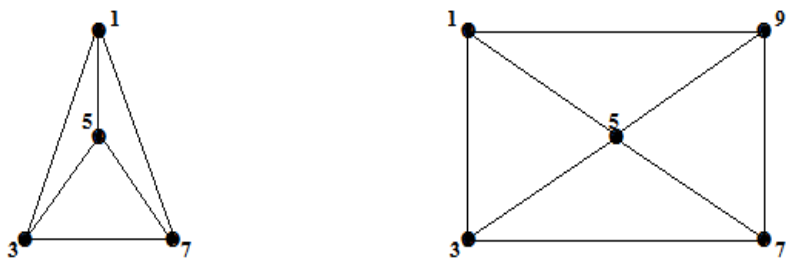

Figure:8

If $n>4$, We have repetition of edge labels, which is not possible. Hence to get the edge label 1 , We need a vertex $u$ with label 1 . There are four more vertices incident with $u$. This is not possible by remark 1.3[5].

Hence $W_{n}, n>4$ is not a Heronian mean graph.

\section{Conclusion}

All graphs are not Heronian mean graphs. In this paper, we proved some more graphs which admits Heronian Mean Graphs. It is very interesting to investigate graphs which admits Heronian Mean Labeling. The derived results are demonstrated by means of sufficient illustrations which provide better understanding. It is possible to investigate more and more graphs and find results which admits Heronian Mean Labeling.

\section{ACKNOWLEDGEMENT}

The authors are thankful to the referee for their valuable comments and suggestions.

\section{REFERENCES}

[1] Harary.F (1988), Graph Theory, Narosa publishing House, New Delhi.

[2] J.A.Gallian, A Dynamic Survey of Graph Labeling. The Electronic Journal of combinatorics(2013).

[3] S.Somasundaram and R.Ponraj, "Mean Labeling of graphs", National Academy of Science Letters vol.26, p.210-213.

[4] S.Somasundaram, R.Ponraj and S.S.Sandhya, "Harmonic Mean Labeling of Graphs", communicated to Journal of Combinatorial Mathematics and Combinatorial Computing.

[5] S.S.Sandhya, E.Ebin Raja Merly and S.D.Deepa, "Heronian Mean Labeling of Graphs", communicated to International Journal of Mathematical Form.

[6] S.S.Sandhya, E.Ebin Raja Merly and S.D.Deepa, "Some Results on Heronian Mean Labeling of Graphs", communicated to Journal of Discrete Mathematical Sciences and Cryptography. 